\title{
Balancing the Intestinal Microbiota to Treat Lower Gastrointestinal Diseases
}

\author{
Chi Ma1, Jianping Hui' \\ 1Shaanxi University of Traditional Chinese Medicine, Xianyang 712046, Shaanxi Province, China \\ ${ }^{2}$ Affiliated Hospital of Shaanxi University of Traditional Chinese Medicine, Xianyang 712000, Shaanxi Province, China \\ *Corresponding author: Jianping Hui, 546893348@qq.com
}

\begin{abstract}
Bacterial colonization is established by direct contact with maternal microorganisms immediately after birth, and may be affected during lactation. Increasing evidence shows that changes in the quantity and quality of the intestinal microbiota can help change the mucosal activation of the immune system, leading to intestinal or extraintestinal diseases. Throughout childhood and adolescence, the balance between pathogenic microbiota and beneficial microbiota is essential for gastrointestinal health, including the protection of pathogens, inhibition of pathogens, nutrient processing (vitamin $\mathrm{K}$ synthesis), and blood vessels where the stimulation of production and the regulation of host fat storage. Probiotics can promote the deliberate regulation of the intestinal microbiota, which is beneficial to the health of the host. The regulatory effects of intestinal microbiota on the prevention and adjuvant treatment of certain gastrointestinal diseases are reviewed.
\end{abstract}

Keywords: Microbiota; Gastrointestinal diseases; Probiotics

Publication date: July 2021; Online publication: July 31, 2021

\section{Introduction}

In recent years, extensive efforts have been made in research and clinical trials to fully understand the intestinal microbiotas' function, development and role in the pathophysiology of certain diseases.

The human intestine is the natural habitat of diverse and dynamic microbial populations, mainly adapted to bacteria living on the mucosal surface of the gastrointestinal cavity. The intestinal microbiota includes natural species that permanently settle in the gastrointestinal tract, as well as a series of constantly changing living microorganisms that pass through the digestive tract through microorganisms. The microbial population of the human gut includes approximately 100 billion bacteria in 500-1000 different species. As part of the intestinal microbiota, certain bacteria are essential for proper nutrition and development of the body and proper maintenance of the immune system. Intestinal homeostasis is essential for efficiently extracting energy from nutrients and preventing microbial pathogens. Experiments on mice have shown that sterile animals (animals without or very minimal amounts of microbiota) are not only smaller than mice that have a normal microbiota at birth or have been recolonized after being sterile, but also have less body fat (leaner). Therefore, it has been observed that normal microbiota or recolonized mice store more fat and eat less food. In addition, sterile mice consume less oxygen.

A thorough understanding of the intestinal microbiota is essential to determine the role of this change in the development of certain diseases. Many diseases are largely caused by changes in the intestinal microbiota, such as infectious diarrhea and diarrhea associated with antibiotic management, sepsis complications (multiple system organ failure, diverticulitis, appendicitis, etc.), functional stomach intestinal diseases (constipation, irritable bowel syndrome, etc.), obesity, type 2 diabetes, metabolic syndrome, autoimmune diseases (including celiac disease) or colon cancer. 
We set out to examine the major pediatric gastrointestinal diseases most closely related to the gastrointestinal microbiota. These include Helicobacter pylori (H. pylori) infection, necrotizing enterocolitis, enteritis, celiac disease and acute diarrhea.

\section{Helicobacter Pylori Infection}

More than $50 \%$ of the world's population are infected with H. pylori. The prevalence rate is $30-40 \%$ in developed countries and more than $80 \%$ in developing countries. Among people under the age of 20, the prevalence of $\mathrm{H}$. pylori is about $80 \%$ in developing countries, which is higher than that in developed countries. The change in prevalence is related to sociodemographic factors, especially among people with low socioeconomic statuses.

Although the bacterial load in the stomach is very low, H. pylori has received special attention due to its association with various gastric diseases. $\mathrm{H}$. pylori is a gram-negative spirochete and is one of the most common bacterial infection drugs in humans. It can cause gastritis, gastric and duodenal ulcers, gastric cancer, adenocarcinoma, and mucosal-related lymphoid tissues. Lymphoma, such as MALT lymphoma. In the absence of ulcer disease, most infections of $\mathrm{H}$. pylori are asymptomatic, and when symptoms are present, they tend to be non-specific. The latest research in adults shows that $\mathrm{H}$. pylori infection increases the risk of coronary heart disease.

There is public evidence that lactic acid bacteria, bifidobacteria, fermented milk, Lactobacillus salivarius and Lactobacillus acidophilus can alleviate the adverse reactions associated with the eradication of H. pylori. Several studies have shown the efficacy of certain probiotics (for example, Bleomyces cerevisiae alone or in combination with Lactobacillus casei) in eradicating $\mathrm{H}$. pylori in pediatric patients. In the adult population, there is more evidence to support this recommendation. Probiotic therapy improves the eradication rate of helicobacter pylori ${ }^{[1]}$.

\section{Necrotizing enterocolitis}

Necrotizing enterocolitis (NEC) is the most common gastrointestinal medical emergency in newborns, and its pathogenesis is unknown. Many factors may lead to its development, such as premature birth, hypoxia, formula feeding, especially excessive protein substrates in the intestinal lumen, sepsis, intestinal ischemia, and colonization of the intestine by pathogens. A recently published study reported that, consistent with other evidence, the proportion of Proteus is higher among newborns diagnosed with NEC. In this study of 9 NEC neonates and 9 neonates in the control group, a lower proportion of these bacteria was observed in NEC patients 1 week before diagnosis compared to the control group. The author points out that premature babies are not colonized by enough bacteria. The bacteria in the first week of life may not be able to regulate the adaptive immune response to subsequent bacterial reproduction. Therefore, the immaturity of the immune tolerance mechanism affected by the quantity and quality of the microbiota may be related to the disease.

\section{Celiaca}

Celiac disease (CD) is currently defined as an immune-mediated systemic disease caused by the intake of gluten in genetically susceptible populations. It is characterized by a variety of gluten-dependent clinical manifestations, CD-specific antibodies, and haplotypes (HLA) DQ2 or HLA DQ8 and gluten excluded from the diet are related to the disappearance of symptoms and CD-specific antibodies in most patients and the normalization of the intestinal mucosa ${ }^{[2]}$.

Studies conducted in recent years have found that the prevalence of CD is 1:100 (range 0.5-2\%). The disease affects children as well as adults, and the ratio of men to women is 2:1.

In patients with celiac disease, the immune response to gliadin fragments mainly causes inflammation 
in the upper small intestine, which is characterized by atrophy of inflammatory cells and villi that infiltrate the lamina propria and epithelium. This response is mediated by innate and adaptive immunity. The adaptive response is mediated by $\mathrm{T}$ CD4+ lymphocytes in the lamina propria that recognize gliadin peptides, which bind to class II HLA molecules (DQ2 or DQ8) expressed in antigen presenting cells. Then, T cells produce pro-inflammatory cytokines, especially interferon gamma. The enzyme tissue transglutaminase catalyzes the deamidation of gliadin peptides in the intestine and enhances its immunogenicity ${ }^{[3]}$.

It is believed that in addition to immune and genetic factors, environmental factors, such as infancy eating patterns and gut microbiota, also play an important role in the pathogenesis of CD. The importance of the microbiota in $\mathrm{CD}$ has yet to be determined. Certain genetic factors are related to the colonization of bacteroides. Some studies conducted on patients with celiac disease have shown that a strict gluten-free diet promotes a reduction in the colonization of beneficial bacteria, compared with the colonization of gramnegative bacteria (E. coli and etc.), especially bifidobacterial milk and bacillus. This change in the microbiota after gluten elimination may be due to the elimination of an important source of carbohydrates, which are the main source of energy, and dietary treatment for these patients cannot promote intestinal homeostasis. However, the immunosuppressive effects of this microbiota may be beneficial to patients with $\mathrm{CD}$. The ongoing research on the role of specific components of the microbiota in the pathogenesis of CD may lead to the development of nutritional intervention strategies (probiotics and prebiotics) for prevention or treatment purposes in the future.

\section{Summary}

The human intestinal microbiota itself constitutes a universe, exists in our body, and has an important influence on our physiology and pathophysiology. The main functions performed by the human intestinal microbiota, such as metabolic functions (fermentation of indigestible dietary substrates and endogenous mucus), protective functions (barriers against pathogens) and nutritional functions (controlling the proliferation and differentiation of epithelial cells). Its role in the development of the immune system and homeostasis allows us to say that the intestinal microbiota is a metabolic organ. An in-depth understanding of the intestinal microbiota is essential to clarify the role of changes in this level in relation to the development of certain intestinal and extra-intestinal diseases.

\section{Disclosure statement}

The author declares no conflict of interest.

\section{References}

[1] Palm NW, Zoete MR, Flavell RA, 2015, Immune-Microbiota Interactions in Health and Disease. Clin Immunol, 159:122-127.

[2] Zhang ZY, Liang T, Li YS, et al., 2019, The Influence of Intestinal Microbiota on Lower Digestive Tract Diseases. Foreign Medicine (Medical Geography), 40, 161(03):124-127.

[3] Wei X, Yu HG, 2020, Adjusting the Intestinal Microbiota to Treat Lower Gastrointestinal Diseases. Medical Review, 26(12):51-55. 